\title{
Pyroelectricity of Molecular Crystals: Benzene Derivatives
}

\author{
Tetsuo Asaji and Alarich Weiss \\ Institut für Physikalische Chemie, Physikalische Chemie III, Technische Hochschule Darmstadt
}

Z. Naturforsch. 40 a, 567-574 (1985); received March 6, 1985

\begin{abstract}
The pyroelectric coefficient at constant stress was measured for meta-nitroaniline, meta-aminophenol, and 2,3-dichlorophenol in the temperature range $90 \leqq T / \mathrm{K} \geqq$ melting point. A change in sign of the pyroelectric coefficient was observed in meta-nitroaniline and 2,3-dichlorophenol. At room temperature $p_{3}^{\sigma}$ is $9 \mu \mathrm{Cm}^{-2} \mathrm{~K}^{-1}$ for meta-nitroaniline, $42 \mu \mathrm{Cm}^{-2} \mathrm{~K}^{-1}$ for meta-aminophenol, and $0.3 \mu \mathrm{Cm}^{-2} \mathrm{~K}^{-1}$ for 2,3-dichlorophenol (at $263 \mathrm{~K}$ for the latter one). By a classical harmonic oscillator model the contribution to the pyroelectric coefficient due to molecular dipole librational motions was estimated to be fairly small for meta-nitroaniline. The temperature dependence of the coefficient of meta-nitroaniline is discussed on the basis of Boguslawski's theory. The importance of internal polar optical modes for the temperature dependence of the pyroelectric coefficient of molecular crystals is shown.
\end{abstract}

\section{Introduction}

Pyroelectricity has gained much attention in the last two decades because of the interest in pyroelectric materials in applied science. Furthermore much work is devoted to the nonlinear optical properties of noncentrosymmetric polar crystals. The majority of studies have been focussed to the pyroelectricity of inorganic compounds or organic salts because in these groups of solids some members show a very large pyroelectric coefficient, a property important in application, e.g. for thermal detectors.

Many molecular solids crystallize with a noncentrosymmetric polar crystal structure. However, their physical properties have not been much explored, probably because of low melting points and weak mechanical strength. A theory of the pyroelectric effect, based on nonclassical physics was first presented by Boguslawski [1]. He assumes that one atomic point position in the pyroelectric crystal performs major anharmonic thermal oscillations. With this assumption the temperature dependence of the primary pyroelectric coefficient can be expressed by the Einstein function for an oscillator system.

With a slight extension of the Boguslawski model the temperature dependence of the primary pyroelectric coefficient of $\mathrm{LiTaO}_{3}$ and $\mathrm{LiSO}_{4} \cdot \mathrm{H}_{2} \mathrm{O}$ was explained and the importance of the polar optical lattice-vibrational modes recognized $[2,3]$. It was

Reprint requests to Prof. Dr. A. Weiss, Institut für Physikalische Chemie, Physikalische Chemie III, Technische Hochschule Darmstadt, Petersenstr. 20, D-6100 Darmstadt, West Germany. found that in these essentially ionic crystals the anharmonicity of the polar optical lattice modes dominates the primary pyroelectric coefficient.

In case of polar molecular crystals with (per definitionem) permanent electric dipole moment, it is expected that the anharmonicity of the low-frequency internal modes and the averaging effect of librational motions of the molecule on the dipole moment take also an important part in the pyroelectric coefficient.

The temperature dependence of the pyroelectric coefficient in molecular crystals has been analyzed for saccharose [4] and $\alpha$-resorcinol (meta-dihydroxybenzene) [5]. Both solids are molecular crystals with such a structure that intermolecular interactions via hydrogen bonds are very likely. The discussion of pyroelectricity in saccharose is limited to the lowtemperature behaviour in which the internal modes contribute negligibly to the pyroelectric coefficient. For $x$-resorcinol an analytical fitting of the pyroelectric coefficient was given for the range $5 \leqq T / \mathrm{K} \leqq 350$. One of the optical frequencies (wave number $\tilde{v} \approx 520 \mathrm{~cm}^{-1}$ ) found from this fitting agrees with that of a strong infrared band at $\tilde{v}=$ $543 \mathrm{~cm}^{-1}$. This band has $A_{1}$ symmetry (polar) and it can be assigned to the substituent sensitive 6 a-mode of meta-disubstituted benzenes [6].

In order to obtain further information about the microscopic mechanism of the temperature dependence of the spontaneous polarization in pyroelectric molecular crystals, the pyroelectric coefficient of three benzene derivatives was studied in the temperature range $90 \leqq T / K \cong$ melting points; this is 
reported here. The compounds are meta-nitroaniline, meta-aminophenol, and 2,3-dichlorophenol, the crystals of them belong to the space groups $\mathrm{C}_{2 \mathrm{v}}^{5}-\mathrm{Pbc} 2_{1}, \mathrm{C}_{2 \mathrm{v}}^{5}-\mathrm{Pca} 2_{1}$, and $\mathrm{C}_{3}^{2,3}-\mathrm{P} 3_{1,2}$, respectively, in the same order $[7-10]$. A positive pyroelectric effect was observed in meta-nitroaniline [11]. However, no numerical values have been reported. For meta-aminophenol Myzgin et al. [12] give a pyroelectric coefficient of $\sim 30 \mu \mathrm{Cm}^{-2} \mathrm{~K}^{-1}$ at arbitrary temperature.

\section{Experimental}

\section{Material}

The starting material for crystal growth of the three compounds investigated was from commercial sources. The chemicals were of purity $\gtrsim 98 \%$ and they were further purified during the crystallization procedures.

Large single crystals of meta-nitroaniline $\left(\mathrm{NO}_{2}\right)^{(3)}\left(\mathrm{NH}_{2}\right)^{(1)} \mathrm{C}_{6} \mathrm{H}_{4}$, m.p. $=385 \mathrm{~K}$, can be grown from melt [13]. After refining cycles a single crystal of meta-nitroaniline with size of $(5 \times 7 \times 40) \mathrm{mm}^{3}$ was grown by zone melting using a translational speed of $0.9 \mathrm{~mm} \cdot \mathrm{h}^{-1}$. Single crystals of metaaminophenol, $\left(\mathrm{NH}_{2}\right)^{(3)}(\mathrm{OH})^{(1)} \mathrm{C}_{6} \mathrm{H}_{4}$, m.p. $=396 \mathrm{~K}$, were grown from alcoholic solution by slow cooling of the solution and evaporation of the solvent. To prevent oxidation the solution was kept in the dark. Single crystals of $(4 \times 8 \times 10) \mathrm{mm}^{3}$ size were produced by this method within 7 days.

2,3-dichlorophenol, $\mathrm{Cl}^{(2)} \mathrm{Cl}^{(3)}(\mathrm{OH})^{(1)} \mathrm{C}_{6} \mathrm{H}_{3}$ melts at $T=330 \mathrm{~K}$. It was purified by sublimation under reduced pressure, melted and degassed in a glass tube and sealed in vacuo. A cylindrical single crystal of 2,3-dichlorophenol, $13 \mathrm{~mm}$ in diameter and $40 \mathrm{~mm}$ long, was grown by the Bridgman method. The pulling speed was $1.8 \mathrm{~mm} \cdot \mathrm{h}^{-1}$.

\section{Measurement of Pyroelectricity}

To determine the direction of the polar axis with respect to the bulk single crystal was no problem in case of meta-aminophenol. The crystal faces could be identified and plates (001), perpendicular to the polar axis [001], were cut with a wire saw. The different plates used for measurements had an area of $\sim 10 \mathrm{~mm}^{2}$ and were $\approx 1.5 \mathrm{~mm}$ thick.

In case of the two compounds grown from melt, meta-nitroaniline shows pronounced cleavage per- pendicular to [010]. Two plates parallel to [010] and perpendicular to each other were cut and tested by pyroelectric measurements at $300 \mathrm{~K}$. In this way the polar axis, positioned in the plane $(010)$ was determined and then appropriate samples for the measurements were prepared. The samples had a thickness of 0.4 to $1.5 \mathrm{~mm}$ and areas of 9 to $18 \mathrm{~mm}^{2}$.

The test measurements on three crystal plates, cut perpendicularly to each other showed in the case of 2,3-dichlorophenol that the polar axis of the trigonal crystal coincides within the limits of error with the axis of the Bridgman tube. For the measurement of pyroelectricity in this compound plates of $\sim 120 \mathrm{~mm}^{2}$ area and up to $2 \mathrm{~mm}$ thickness were prepared.

The single crystal plates were painted by air drying silver paste on the two opposite faces and inserted between copper electrodes into a cryostat similar to that used by Lang et al. [14]. The temperature at the site of the sample was measured by thermocouples. With this arrangement the pyroelectric voltage was measured in three ways.

a) Pyroelectric coefficient measurement at nearly constant rate of temperature change: The pyroelectric current is measured during the continuous change of the sample temperature by use of a shunt resistance and an electrometer. From the rate of temperature change and the pyroelectric current measured, the pyroelectric coefficient is calculated $[15,16]$. An ultra high input impedance electrometer, Cary model 31, vibrating reed electrometer, was employed using a shunt resistance of $10^{10} \Omega$. The temperature of the sample was varied by $\lesssim 2 \mathrm{~K} \cdot \mathrm{min}^{-1}$ in order to keep the difference between sample temperature and the temperature indicated by the thermocouple at a minimum and also to minimize temperature gradients within the sample.

b) Charge integration: The pyroelectric crystal is connected to a large capacitor (here $10 \mathrm{nF}$ ). From the voltage which appears on the capacitor due to a small change of the crystal temperature the pyroelectric coefficient can be evaluated [16]. For the voltage measurements the same electrometer was used as described under a) but without shunt resistance.

c) Charge integration via operational amplifier: Instead of a capacitor (see b)), a charge integrating amplifier is used to collect the pyroelectric charge released $[16,17]$. The effective input impedance of 
the operational amplifier is very small and therefore essentially short circuit conditions are maintained for the input signal. Hence, the change of the polarization can be monitored continuously as a function of temperature. A low drift integrator was constructed for those measurements incorporating an Analog Devices Operational Amplifier AD $515 \mathrm{~K}$ (maximum input bias current $150 \mathrm{fA}$ ).

d) Pyroelectric voltage measurement under low frequency sinusoidal temperature oscillation: The temperature of the crystal is changed sinusoidally and from the sinusoidal pyroelectric voltage across a shunt resistance the pyroelectric coefficient is calculated. The set up used here was constructed following the circuit given by Hartley et al. [18]. An ultra high impedance operational amplifier (Analog Devices, type $\mathrm{AD} 311 \mathrm{~K}$ ) and a shunt resistance of
$10.5 \mathrm{M} \Omega$ were used. The temperature oscillation was created by a Peltier element driven with a frequency of $0.01-0.02 \mathrm{~Hz}$; the amplitude was $\approx 2 \mathrm{~K}$. In applying this method, silver paste was used to fix the crystal on a copper plate which in turn was connected to the Peltier element.

To suppress sublimation of the materials studied the crystals were held under an atmosphere of dry nitrogen gas. No sublimation was observed and the resistance of the crystals studied was in any case sufficiently high compared to the shunt resistance. The measurements were carried through with increasing and with decreasing temperature to check the existence of nonpyroelectric current sources. The true pyroelectric current reverses its polarity on changing from cooling to heating and vice versa [19].

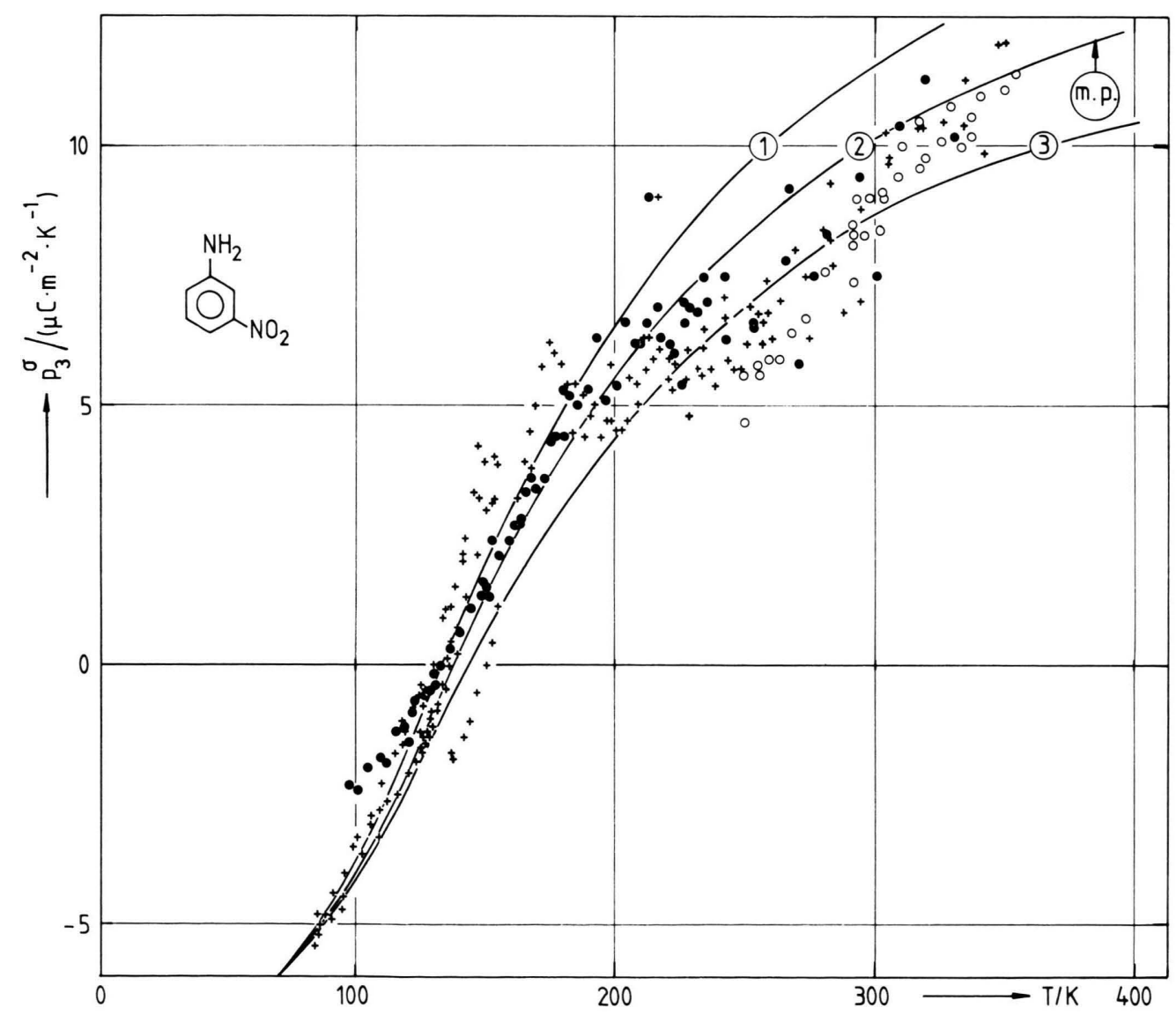

Fig. 1. Temperature dependence of the pyroelectric coefficient $p_{3}^{\sigma}$ of meta-nitroaniline measured at constant stress. - The solid curves 1, 2, and 3 were calculated by means of (11) with $c_{1}=24,22$, and $20 \mu \mathrm{Cm}^{-2} \mathrm{~K}^{-1}$, respectively, and $c_{2}=$ $6.5 \mu \mathrm{Cm}^{-2} \mathrm{~K}^{-1}$, and $\Theta=554 \mathrm{~K}$ (see text). ( ) measured by method (a) and (b), cooling cycle; $(+)$ measured by method (a) and (b), heating cycle; $(O)$ measured by method (d). 


\section{Results}

The pyroelectric coefficient $p_{i}^{\sigma}$ at constant stress $\sigma$ and constant electric field $E$ is defined by

$$
p_{i}^{\sigma}=\left(\frac{\partial D_{i}}{\partial T}\right)_{\sigma, E}=\left(\frac{\partial P_{i}}{\partial T}\right)_{\sigma, E} .
$$

Here, $D_{i}$ and $P_{i}$, denote, respectively, the components of the electric displacement and polarization vectors, and $T$ is the temperature. $p_{i}^{\sigma}$ is the sum of the primary pyroelectric coefficient $p_{i}^{\eta}$ defined at constant strain $\eta$ and constant $E$, and the piezoelectric effect due to thermal strain (the secondary pyroelectric coefficient $\left.p_{i}^{\text {sec }}\right)$. This relationship can be expressed by the tensor equation [20]

$$
p_{i}^{\sigma}=p_{i}^{\eta}+d_{i, j, k}^{T, E} \cdot c_{j, k, l, m}^{T_{1} E_{1, m}} \cdot \alpha_{l, m}^{\sigma, E}=p_{i}^{\eta}+p_{i}^{\mathrm{sec}},
$$

where $d_{i, j, k}^{T, E}$ and $c_{j, k, l, m}^{T, E}$ denote, respectively, the tensor components of the piezoelectric and elastic stiffness moduli measured at constant $T$ and $E$, whereas $\alpha_{l, m}^{\sigma, E}$ is the thermal expansion tensor at constant $\sigma$ and $E$.

The pyroelectric coefficient $\mathbf{p}=\left(p_{1}, p_{2}, p_{3}\right)$ defined above is a vector quantity. The direction of the vector is parallel to the crystal polar axis. The value along the polar axis is denoted by use of subscript letter $i=3$ for the point groups $\mathrm{C}_{2 \mathrm{v}}-\mathrm{mm} 2$ and $\mathrm{C}_{3}-3$ of the present compounds.

No attempt was made in this work to determine the sign of the coefficient $p_{i}^{\sigma}$ [21]. Hence, the sign of the polarization change was not determined.

The pyroelectric coefficients $p_{3}^{\sigma}$ determined are listed for two temperatures in Table 1 and in Fig. 1 the temperature dependence of $p_{3}^{\sigma}$ for meta-nitroaniline is given. The full circles were measured with decreasing temperature and the crosses with increasing temperature on the same crystal and by the

Table 1. Pyroelectric coefficients $p_{3}^{\sigma}$ of three benzene derivatives at two temperatures. The absolute sign of $p_{3}^{\sigma}$ has not been determined.

\begin{tabular}{lcc}
\hline Compound & \multicolumn{2}{c}{$p_{3}^{\sigma} /\left(\mu \mathrm{Cm}^{-2} \mathrm{~K}^{-1}\right)$} \\
\cline { 2 - 3 } & $T=100 \mathrm{~K}$ & $T=295 \mathrm{~K}$ \\
\hline meta-nitroaniline & $-4 \pm 1$ & $9 \pm 1$ \\
meta-aminophenol & $24 \pm 2$ & $42 \pm 2$ \\
2,3-dichlorophenol & $-1.1 \pm 0.1$ & $0.3 \pm 0.1$ \\
& & $(T=263 \mathrm{~K})$ \\
\hline
\end{tabular}

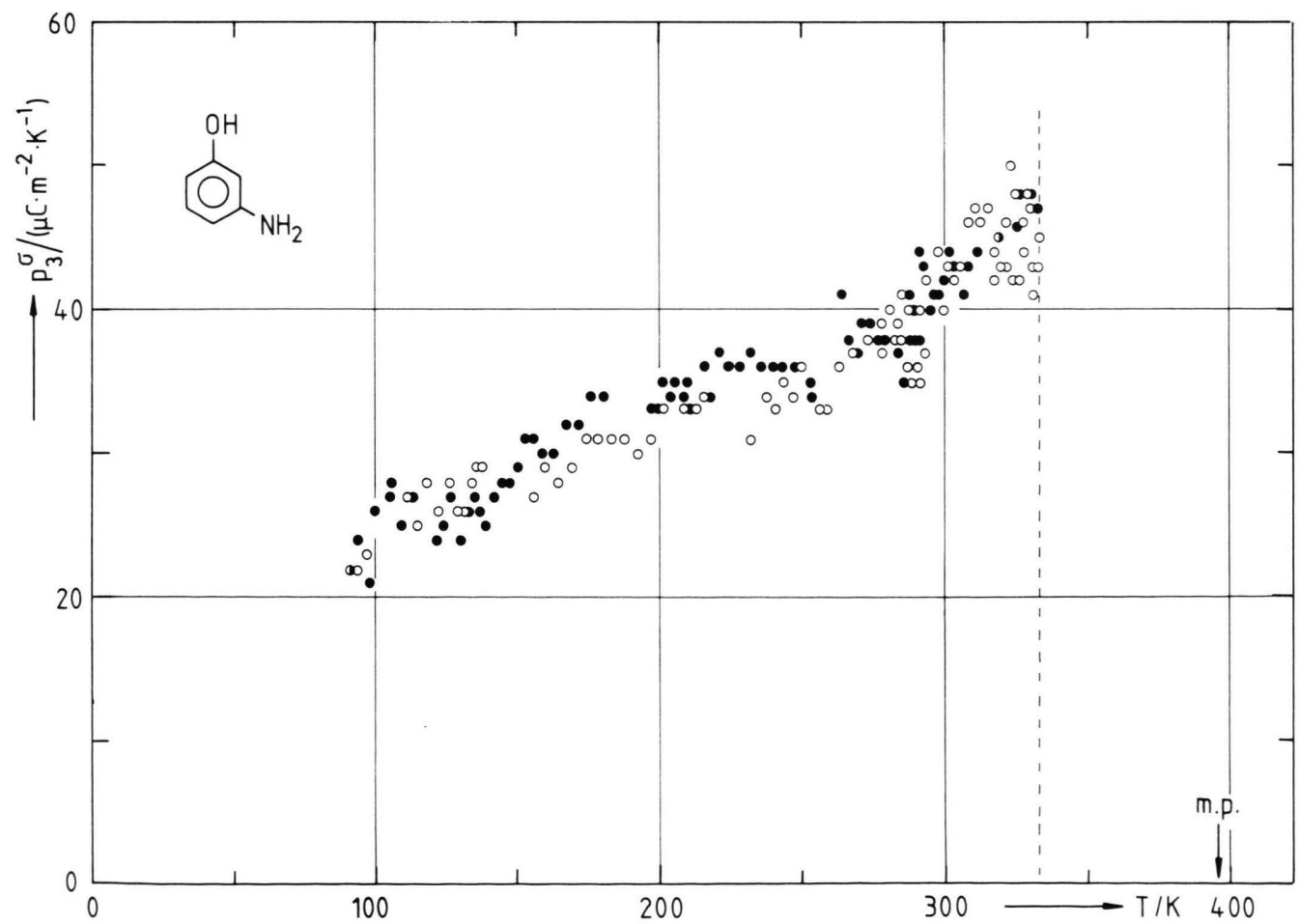

Fig. 2. Temperature dependence of the pyroelectric coefficient $p_{3}^{\sigma}$ of meta-aminophenol measured by the method (a).

$(\bullet)$ Decreasing temperature, $(\bigcirc)$ increasing temperature. 


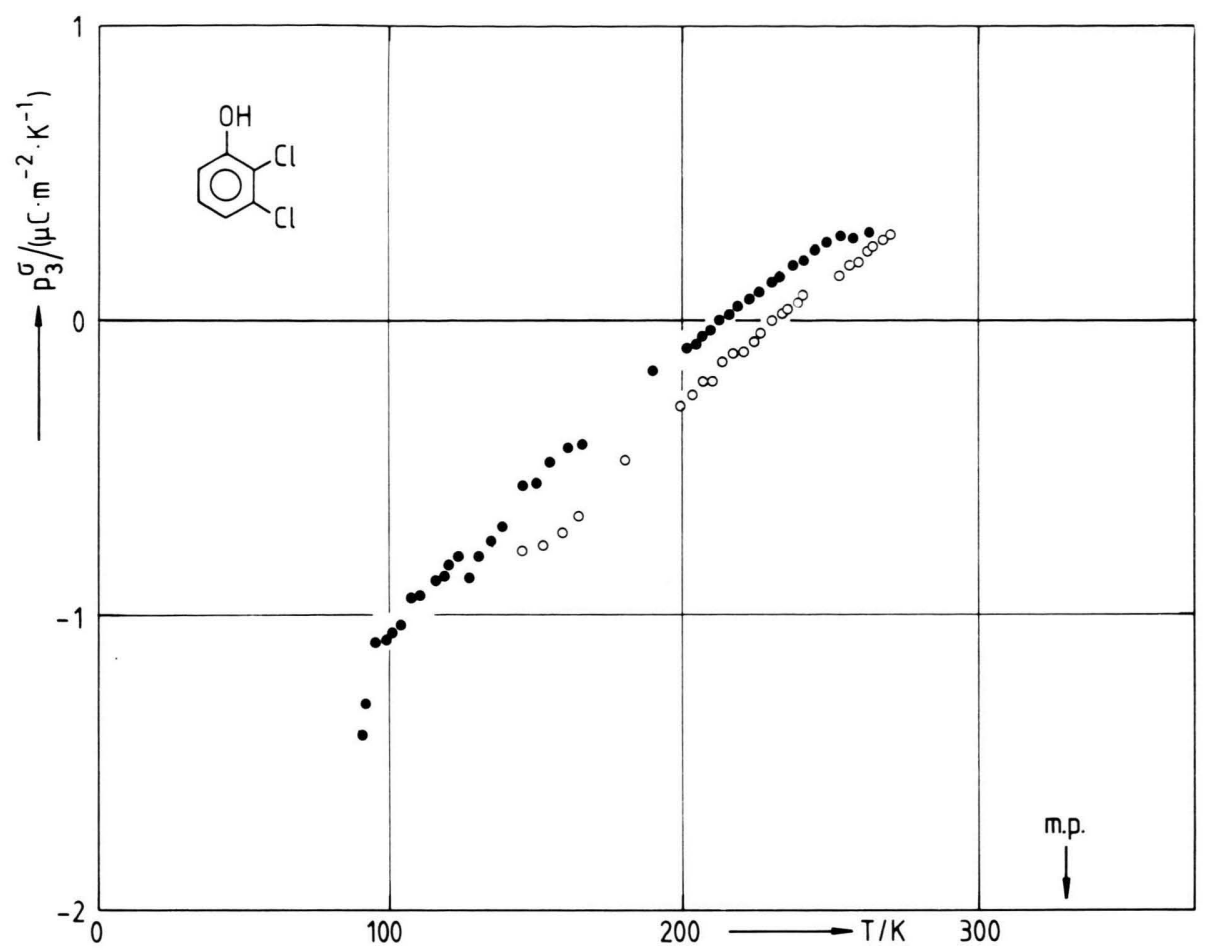

Fig. 3. Temperature dependence of the pyroelectric coefficient $p_{3}^{\sigma}$ of 2,3-dichlorophenol measured by method (a). $(\bullet)$ Decreasing temperature, $(0)$ increasing temperature.

two methods a) and b), while the open circles show the data points obtained by use of sinusoidal temperature oscillation method using a different crystal plate. Above ca. $350 \mathrm{~K}$ an abnormal current was detected which is not of pyroelectric origin, because it was observed at constant temperature. Due to this abnormal current, the pyroelectric coefficient could not be measured beyond this temperature. Sign reversal of the pyroelectric coefficient was observed at about $130 \mathrm{~K}$.

Figures 2 and 3 show the results obtained for meta-aminophenol and 2,3-dichlorophenol, respectively. The measurements at higher temperatures were also limited because of nonpyroelectric current and rapid decrease of resistance of the crystals. A sign reversal of $p_{3}^{\sigma}$, similar to that found for metanitroaniline, was observed in 2,3-dichlorophenol at $T \approx 220 \mathrm{~K}$.

\section{Discussion}

\section{Sign reversal of the pyroelectric coefficient}

The pyroelectric coefficients of meta-nitroaniline and 2,3-dichlorophenol change sign smoothly at about $130 \mathrm{~K}$ and $220 \mathrm{~K}$, respectively. A similar change in sign of the pyroelectric coefficient $p_{i}^{\sigma}$ has been observed in $\mathrm{Ba}\left(\mathrm{NO}_{2}\right)_{2} \cdot \mathrm{H}_{2} \mathrm{O}[22,23], \mathrm{Li}_{2} \mathrm{SO}_{4}$ $\cdot \mathrm{H}_{2} \mathrm{O}$ [3, 22], $\mathrm{KH}_{2} \mathrm{PO}_{4}$ [24], and $\mathrm{NaNO}_{2}$ [25]. For $\mathrm{Ba}\left(\mathrm{NO}_{2}\right)_{2} \cdot \mathrm{H}_{2} \mathrm{O}$ and for $\mathrm{Li}_{2} \mathrm{SO}_{4} \cdot \mathrm{H}_{2} \mathrm{O}$ it was revealed that the primary coefficient itself changes its sign $[3,23]$.

Three possible explanations can be considered for the sign reversal of $p_{i}^{\sigma}$ : (i) phase transformation, (ii) cancellation of primary and secondary coefficient, (iii) mutual cancellation of the opposite contributions from optical modes.

The possibility of a phase transition is excluded for the four inorganic compounds mentioned above. In case of meta-nitroaniline and 2,3-dichlorophenol, where we observed a change of sign in $p_{3}^{\sigma}$, phase transformation near $130 \mathrm{~K}$ and $220 \mathrm{~K}$ respectively, can be excluded, too. For example, the Raman spectra of meta-nitroaniline do not show significant changes by cooling a crystal down to liquid helium temperature [26]. The ${ }^{35} \mathrm{Cl} \mathrm{NQR}$ spectrum of 2,3-dichlorophenol is reported in literature for room temperature and $77 \mathrm{~K}[27,28]$. We have done some measurements of ${ }^{35} \mathrm{Cl}-\mathrm{NQR}$ as a function of tem- 
perature in the range $-75 \leqq T /{ }^{\circ} \mathrm{C} \leqq+25$ and did not find any sign for phase transition. From this we conclude that there is no drastic change of crystal symmetry in the range $77 \leqq T / \mathrm{K} \leqq 298$ and the origin of sign reversal must be due to either cancellation of the primary and secondary pyroelectric coefficient or mutual cancellation of the opposite contributions from optical modes.

\section{Effect of librational motions of molecular dipoles on $p_{3}^{\sigma}$}

The thermal average $\langle\overline{\cos \gamma}\rangle$ of the instantaneous angle $\&$ between the molecular dipole direction and the polar axis of the crystal changes with temperature. Because of the change of librational motions the polarization of the crystal changes too. In $\langle\overline{\cos \gamma}\rangle$ bar and bracket denote, respectively, the average of $\cos \vartheta$ for one energy state and the statistical average of $\overline{\cos \gamma}$ over the energy states at a given temperature.

The pyroelectric coefficient $p_{\text {lib }}$ due to dipole librational motions can be estimated by use of a classical harmonic oscillator model in which the ensemble of molecular oscillators is represented by a classical harmonic oscillator. Mopsik and Broadhurst [29] have shown, that

$$
\frac{\partial(\overline{\cos \vartheta})}{\partial T}=-\cos \vartheta_{0} J_{1}\left(\Phi_{0}\right) \frac{\partial \Phi_{0}}{\partial T},
$$

where $\vartheta_{0}$ is the angle between the polar axis of the crystal and the direction $z^{\prime}$, the average direction of the dipole. $\Phi_{0}$ is the amplitude of a classical rotational harmonic oscillator and the function $J_{1}(x)$ denotes the Bessel function of first kind and first order. In the above expression, only the librational motion about one axis (e.g. $y^{\prime}$ ) perpendicular to the average dipole direction $z^{\prime}$ is considered.

More generally, librational motions about two orthogonal axes, $x^{\prime}$ and $y^{\prime}$, lying in the plane perpendicular to the $z^{\prime}$-axis should be taken into account. Then the following expression is obtained:

$$
\begin{aligned}
\frac{\partial(\cos \vartheta)}{\partial T}= & -\cos \vartheta_{0}\left[J_{0}\left(\Phi_{0, x^{\prime}}\right) J_{1}\left(\Phi_{0, y^{\prime}}\right) \frac{\partial \Phi_{0, y^{\prime}}}{\partial T}\right. \\
& \left.+J_{0}\left(\Phi_{0, y^{\prime}}\right) J_{1}\left(\Phi_{0, x^{\prime}}\right) \frac{\partial \Phi_{0, x^{\prime}}}{\partial T}\right] .
\end{aligned}
$$

Here, $J_{0}(x)$ denotes the Bessel function of first kind and zero order.
The amplitudes $\Phi_{0, x^{\prime}}$ and $\Phi_{0, y^{\prime}}$ of a classical rotational harmonic oscillator about the axes $x^{\prime}$ and $y^{\prime}$, respectively, are defined by the mean-squares amplitude of libration about each axis as

$$
\Phi_{0, i} \equiv\left(2\left\langle\overline{\Phi_{i}^{2}}\right\rangle\right)^{1 / 2} ; \quad i=x^{\prime}, y^{\prime} .
$$

In the classical harmonic approximation, the temperature derivative of the amplitude defined in (5) is given by

$$
\frac{\partial \Phi_{0, i}}{\partial T}=\frac{\Phi_{0, i}}{2 T} ; \quad i=x^{\prime}, y^{\prime} .
$$

Hence, for a crystal consisting of crystallographically equivalent molecules, the pyroelectric coefficient $p_{\text {lib }}$ due to molecular dipole librational motions can be formulated as

$$
\begin{aligned}
p_{\text {lib }}= & \left|\frac{\mu}{V} \frac{\partial(\overline{\cos \bar{\gamma}})}{\partial T}\right|=\left|\frac{\mu}{V} \frac{\cos \vartheta_{0}}{2 T}\right| . \\
& \cdot\left[J_{0}\left(\Phi_{0, x^{\prime}}\right) J_{1}\left(\Phi_{0, y^{\prime}}\right) \Phi_{0, y^{\prime}}\right. \\
& \left.+J_{0}\left(\Phi_{0, y^{\prime}}\right) J_{1}\left(\Phi_{0, x^{\prime}}\right) \Phi_{0, x^{\prime}}\right],
\end{aligned}
$$

where $\mu$ and $V$ denote the electric dipole moment of the molecule and the volume per molecule, respectively. In case the unit cell contains more than one crystallographically nonequivalent molecule, (7) gives the contribution of one of these molecules, and the individual contributions have to be added before taking the absolute value in estimating the magnitude of $p_{\text {lib }}$.

For meta-nitroaniline, the mean-squares amplitudes of librational motions about the principal axes of moments of inertia can be evaluated by using the Raman spectroscopic data on lattice vibrations and the tensor of moments of inertia given by Szostak [30]. If $I_{i}$ are the moments of inertia of the molecule and $\omega_{i}$ its angular frequencies of librational motion about the principal axes system with the coordinates $u, v, w$, by classical approximation one finds

$$
\left\langle\overline{\Phi_{i}^{2}}\right\rangle=\frac{k T}{I_{i} \omega_{i}^{2}} ; \quad i=u, v, w .
$$

Using the formalism given by Cruickshank [31] we have calculated from the data of Szostak the rotational amplitudes around $x^{\prime}, y^{\prime}, z^{\prime}$. Thereby the $y^{\prime}-$ axis was defined in the plane $w z^{\prime}$. The direction $z^{\prime}$ of the average position of the molecular dipole and its magnitude were evaluated by summing up the partial dipole moments of the substituent groups 
[32] in the molecule and by referring to the coordinates determined in the X-ray structure analysis [8]. In this way the following values have been found for meta-nitroaniline at $T=250 \mathrm{~K}$ : Molecular dipole moment $\mu=1.65 \cdot 10^{-29} \mathrm{Cm} ; \vartheta_{0}=51^{\circ} ; V=$ $16 \cdot 10^{-29} \mathrm{~m}^{3} ; \Phi_{0, x^{\prime}}=0.07 \quad(T=250 \mathrm{~K}) ; \Phi_{0, y^{\prime}}=0.11$ $(T=250 \mathrm{~K})$. Then, from (7) $p_{\text {lib }}$ is estimated to $1 \mu \mathrm{Cm}^{-2} \mathrm{~K}^{-1}$ at $250 \mathrm{~K}$. In comparison, the observed value of $p_{3}^{\sigma}$ is $6 \mu \mathrm{Cm}^{-2} \mathrm{~K}^{-1}$ at $250 \mathrm{~K}$, varying from $-5 \mu \mathrm{Cm}^{-2} \mathrm{~K}^{-1}$ to $+10 \mu \mathrm{Cm}^{-2} \mathrm{~K}^{-1}$ on changing the temperature from $90 \mathrm{~K}$ to $310 \mathrm{~K}$.

From this estimate we conclude that the averaging effect of molecular dipoles by librational motions contributes little to the observed temperature variation of $p_{3}^{\sigma}$ in meta-nitroaniline.

For the two other compounds studies here, metaaminophenol and 2,3-dichlorophenol, there is no report in literature about the librational motions and an estimate of $p_{\text {lib }}$, as done for meta-nitroaniline, is not possible. However, that factor in (7), which is independent of the amplitude of librational motions (the factor $\left.\frac{\mu}{V} \frac{\cos \vartheta_{0}}{2 T}\right)$ was estimated for meta-aminophenol and for 2,3-dichlorophenol by use of the known crystal structure data and partial dipole moments [32]. The partial dipole moment of the bond $\mathrm{C}-\mathrm{Cl}$ was taken from $\mathrm{LB}$, Vol. I/3, p. 405 (1951): $\mu_{\mathrm{C}-\mathrm{Cl}}=1.6$ Debye. It was found that $p_{\text {lib }}$, calculated in this way is about $40 \%$ (metaaminophenol) and $13 \%$ (2,3-dichlorophenol) of $p_{\text {lib }}$ found for meta-nitroaniline. It has to be mentioned that in 2,3-dichlorophenol there are 3 molecules in the asymmetric unit of the unit cell, and one of the coefficients $\frac{\mu}{V} \frac{\cos \vartheta_{0}}{2 T}$ has the opposite sign of the other two. The estimate of $p_{\text {lib }}$ for meta-aminophenol shows that the librational effect can hardly be responsible for $p_{3}^{\sigma}$ since the ratio $p_{3}^{\sigma} / p_{\text {lib }}$ is here 10 times larger than in meta-nitroaniline.

It seems that librational motions contribute little to the pyroelectric coefficient in molecular crystals. We confirmed this by carrying out similar calculations for $\alpha$-resorcinol on the basis of the known mean squares amplitudes of librational motions deduced from a neutron diffraction analysis [33]. $p_{\text {lib }}=0.5 \mu \mathrm{Cm}^{-2} \mathrm{~K}^{-1}$ was found at $T=250 \mathrm{~K}$, a value which is two orders of magnitude smaller than $p_{3}^{\sigma}$ found from experiment. $p_{3}^{\sigma}$ of $\alpha$-resorcinol at room temperature is reported by different authors to be $26 \mu \mathrm{Cm}^{-2} \mathrm{~K}^{-1}$ and $120 \mu \mathrm{Cm}^{-2} \mathrm{~K}^{-1}[5,34,35]$.
Contribution to $p_{3}^{\sigma}$ originating from polar optical modes

As stated above, the librational motions effect is a rather minor contribution to $p_{3}^{\sigma}$ and to the temperature dependence of $p_{3}^{\sigma}$. In the following we shall try to explain $p_{3}^{\sigma}=f(T)$ of meta-nitroaniline by a theoretical expression based on Boguslawski's prediction [1]. According to $[2,3] p_{3}^{\eta}$ is given by

$$
p_{3}^{\eta}=\sum_{i} c_{i} E\left(\Theta_{i} / T\right),
$$

$c_{i}$ being the coefficients which describe the relative amplitude of each contributing polar mode $i$, and $\Theta_{i}$ being the Einstein temperatures defined by $h v_{i} / k$ for the mode $i$ with frequency $v_{i}$. The Einstein function $E(x)$ is given by

$$
E(x)=x^{2} e^{x} /\left(e^{x}-1\right)^{2} .
$$

In case of meta-nitroaniline, for which compound the vibrational data are available, the relation between the pyroelectric coefficient and the Einstein temperature can be applied under certain assumptions. We assume that the anharmonicity of a single polar optical mode predominates the temperature dependence of $p_{3}^{\sigma}$. Then (9) can be replaced by

$$
p_{3}^{\sigma}=c_{1} E(\Theta / T)-c_{2} .
$$

The coefficient $c_{2}$ includes all other contributions, i.e. the remaining polar modes and the secondary effect; $c_{2}$ is assumed to be practically independent of temperature above $T=90 \mathrm{~K}$. Equation (11) can be fitted to the experimental data.

As reported by Szostak [30], all lattice vibrational modes of meta-nitroaniline can be assigned to bands lying below $150 \mathrm{~cm}^{-1}$. The Einstein temperatures corresponding to these frequencies cannot explain the increase of $p_{3}^{\sigma}$ observed at room temperature.

On investigating the IR spectrum of meta-nitroaniline with polarized radiation $(\boldsymbol{E} \| c)$ two strong bands are observed at $\tilde{v}=385 \mathrm{~cm}^{-1}$ and $\tilde{v}=540 \mathrm{~cm}^{-1}$, and furthermore, with unpolarized radiation bands at $\tilde{v}=227 \mathrm{~cm}^{-1}, \tilde{v}=383 \mathrm{~cm}^{-1}$, and $\tilde{v}=541 \mathrm{~cm}^{-1}$ are found [26]. The Einstein temperatures corresponding to the wave numbers $227 \mathrm{~cm}^{-1}, 385 \mathrm{~cm}^{-1}$, and $540 \mathrm{~cm}^{-1}$ are $327 \mathrm{~K}, 554 \mathrm{~K}$, and $777 \mathrm{~K}$, respectively. For the fact that $p_{3}^{\sigma}$ is still increasing at room temperature, $\Theta=327 \mathrm{~K}$ cannot account for; it is not high enough. Using the Einstein temperatures $\Theta=554 \mathrm{~K}$ and $777 \mathrm{~K}$, one finds a maximum for $\mathrm{d} E(\Theta / T) / \mathrm{d} T$ at $122 \mathrm{~K}$ and $171 \mathrm{~K}$, respectively. 
In the experimental curve $p_{3}^{\sigma}$ vs. $T$ (Fig. 1) with raising temperature the slope starts to decrease clearly above $170 \mathrm{~K}$. The mode at $540 \mathrm{~cm}^{-1}$ corresponding to $\Theta=777 \mathrm{~K}$ would shift the temperature, at which a decrease of the slope $p_{3}^{\sigma}=f(T)$ is clearly observed, to $T>170 \mathrm{~K}$. Hence we conclude that $\Theta=554 \mathrm{~K}\left(\tilde{v}=385 \mathrm{~cm}^{-1}\right)$ is the important Einstein temperature (vibration) for $p_{3}^{\sigma}$. With $c_{1}=$ $22 \mu \mathrm{Cm}^{-2} \mathrm{~K}^{-1}, \quad c_{2}=6.5 \mu \mathrm{Cm}^{-2} \mathrm{~K}^{-1}$, and $\Theta=554 \mathrm{~K}$ the pyroelectric coefficient $p_{3}^{\sigma}$ measured for metanitroaniline is well described by (11) throughout the whole temperature range investigated. This is shown in Figure 1.

This result suggests the importance of internal modes in the temperature dependence of the pyroelectric coefficient of molecular crystals. GarrigouLagrange et al. [36] have assigned the polar mode of meta-nitroaniline o.bserved at $385 \mathrm{~cm}^{-1}$ to the substituent (X) sensitive mode $\tilde{v}_{\mathrm{c}}$ of Kohlrausch [37]. This mode, corresponding to the $6 \mathrm{a}$-mode in Wil-

[1] S. Boguslawski, Phys. Z. 15, 569 (1914); ibid 805 (1914).

[2] M. E. Lines and A. M. Glass, Phys. Rev. Lett. 39, 1362 (1977).

[3] S. B. Lang, Phys. Rev. B4, 3603 (1971).

[4] J. Mangin and A. Hadni, Phys. Rev. B 18, 7139 (1978).

[5] N. D. Gavrilova, S. N. Drozhdin, V. K. Novik, and E. G. Maksimov, Solid State Commun. 48, 129 (1983).

[6] G. N. R. Tripathi, J. Chem. Phys. 74, 250 (1981).

[7] J. L. Stevenson and A. C. Skapski, J. Phys. C: Solid State Phys. 5, L 233 (1972).

[8] A. C. Skapski and J. L. Stevenson, J. C. S. Perkin II, $1973,1197$.

[9] C. de Rango, S. Brunie, G. Tsoucaris, J. P. Declercq, and G. Germain, Cryst. Struct. Commun. 3, 485 (1974).

[10] C. Bavoux and A. Thozet, Cryst. Struct. Commun. 5, 259 (1976).

[11] N. D. Gavrilova, Sov. Phys. Crystallogr. 10, 91 (1965).

[12] E. A. Myzgin, B. A. Chayanov, L. A. Beresnev, and L. M. Blinov, Sov. Phys. Crystallogr. 27, 126 (1982).

[13] A. Carenco, J. Jerphagnon, and A. Perigaud, J. Chem. Phys. 66, 3806 (1977).

[14] S. B. Lang, S. A. Shaw, L. H. Rice, and K. D. Timmerhaus, Rev. Sci. Instrum. 40, 274 (1969).

[15] R. L. Byer and C. B. Roundy, Ferroelectrics 3, 333 (1972).

[16] S. B. Lang, Sourcebook of Pyroelectricity, pp. 32, Gordon and Breach Science Publishers, New York 1974.

[17] A. M. Glass, J. Appl. Phys. 40, 4699 (1969).

[18] N. P. Hartley, P. T. Squire, and E. H. Putley, J. Phys. E5, 787 (1972).

[19] J. P. Dougherty and R. J. Seymour, Rev. Sci. Instrum. 51, 229 (1980).

[20] J. F. Nye, Physical Properties of Crystals, Chapt. X, Clarendon, Oxford 1957.

[21] Landolt-Börnstein, New Series, Vol. III/18, p. 326, Springer-Verlag, Heidelberg 1984. son's notation [38], is a radial skeletal vibration strongly coupled to the $\mathrm{C}-\mathrm{X}$ stretching vibration [39]. Although Szostak [26] presented another assignment for the same band, it can be assigned to a vibrational mode with appreciable amplitude of the substituents $X$. Its anharmonicity must be quite strong to account for reasonably large deformations of the molecular structure by changing the temperature. This effect in turn then is responsible for the temperature dependence of $p_{3}^{\sigma}$.

To confirm the estimates presented here, the temperature dependence of the primary coefficient $p_{3}^{\eta}$ should be analyzed and a precise structure analysis at various temperatures is required. Such efforts have been presented for $\mathrm{Ba}\left(\mathrm{NO}_{2}\right)_{2} \cdot \mathrm{H}_{2} \mathrm{O}$ [40], Tourmaline [41] and $\mathrm{Li}_{2} \mathrm{SO}_{4} \cdot \mathrm{H}_{2} \mathrm{O}$ [42] to correlate the temperature dependence of atomic positions with observed polarization changes.

We are grateful to the Stiftung Volkswagenwerk for support of this work.

[22] V. V. Gladkii and I. S. Zheludev, Sov. Phys. Crystallogr. 10,50 (1965).

[23] V. V. Gladkii and I. S. Zheludev, Sov. Phys. Crystallogr. 12, 788 (1968).

[24] S. Vieira, C. de las Heras, and J. A. Gonzálo, Solid State Commun. 31, 175 (1979).

[25] C. de las Heras, J. A. Gonzálo, and S. Vieira, Ferroelectrics 33, 13 (1981).

[26] M. M. Szostak, J. Raman Spectrosc. 8, 43 (1979).

[27] W. Pies and Al. Weiss, in J. A. S. Smith (Ed.), Advances in Nuclear Quadrupole Resonance, Vol. 1, Heyden, London 1974, p. 57.

[28] J. P. Bayle, J. Jullien, H. Stahl-Lariviere, and L. Guibe, J. Mol. Struct. 58, 487 (1980).

[29] F. I. Mopsik and M. G. Broadhurst, J. Appl. Phys. 46, 4204 (1975).

[30] M. M. Szostak, J. Raman Spectrosc. 12, 228 (1982).

[31] D. W. J. Cruickshank, Acta Crystallogr. 9, 754 (1956).

[32] J. G. Bergman and G. R. Crane, J. Chem. Phys. 66, 3803 (1977).

[33] G. E. Bacon and R. J. Jude, Z. Kristallogr. 138, 19 (1973).

[34] F. Hayashi, Dissertation, Göttingen 1912, Data quoted in International Critical Tables, Vol. VI, pp. $207-212$.

[35] V. A. Koptsik, Sov. Phys. Crystallogr. 4, 197 (1960).

[36] C. Garrigou-Lagrange, M. Chehata, and J. Lascombe, J. Chim. Phys. (Paris) 63, 552 (1966).

[37] K. W. F. Kohlrausch, Phys. Z. 37, 58 (1936).

[38] E. B. Wilson, Jr., Phys. Rev. 45, 706 (1934).

[39] G. Varsányi, Vibrational Spectra of Benzene Derivatives, Academic Press, New York 1969, pp. 256-259.

[40] R. Liminga, S. C. Abrahams, A. M. Glass, and A. Kvick, Phys. Rev. B 26, 6896 (1982).

[41] G. Donnay, Acta Crystallogr. A33, 927 (1977).

[42] J.-O. Lundgren, Å. Kvick, M. Karppinen, R. Liminga, and S. C. Abrahams, J. Chem. Phys. 80, 423 (1984). 